\title{
Mild TBI/Concussion Clinical Tools for Providers Used Within the Department of Defense and Defense Health Agency
}

\author{
Megan A. Lindberg; Seth A. Kiser, PhD; and Elisabeth M. Moy Martin, RNC, MA
}

\begin{abstract}
Background: Military personnel are at greater risk for sustaining mild traumatic brain injury (mTBI), or concussion, whether they are in a combat or garrison setting. Consequently, mTBI is a major health concern for health practitioners to understand, in order to provide timely assessment and treatment to service members (SM) who are suspected to have mTBI.
\end{abstract}

Observations: Providing early diagnosis and effective management of symptoms can optimize recovery and promote healthy outcomes. Understanding what resources and guidelines are available is important for those evaluating active duty SMs within the Military Health System.

Conclusions: This article showcases clinical tools for screening, evaluating, and diagnosing concussion used within the US Department of Defense, and provides resources for practitioners to find these clinical tools online.

\section{Megan Lindberg is a Research Specialist; Seth Kiser is a Research Scientist; and Elisabeth Moy Martin is the Chief of Clinical Translation; all at Defense and Veterans Brain Injury Center in Silver Spring, Maryland. Megan Lindberg is a Research Specialist at Credence Management Solutions, LLC in Vienna, Virginia. Seth Kiser is a Research Scientist at General Dynamics Information Technology in Falls Church, Virginia. Correspondence: Megan Lindberg (megan.a.lindberg.ctr@ mail.mil)}

Fed Pract. 2020;37(9):410-419. doi:10.12788/fp.0044
T raumatic brain injury (TBI) is a major health concern that can cause significant disability as well as economic and social burden. The Centers for Disease Control and Prevention (CDC) reported a $58 \%$ increase in the number of TBI-related emergency department visits, hospitalizations, and deaths from 2006 to 2014. ${ }^{1}$ In the CDC report, falls and motor vehicle accidents accounted for $52.3 \%$ and $20.4 \%$, respectively, of all civilian TBI-related hospitalizations. In 2014, 56,800 TBIs in the US resulted in death. A large proportion of severe TBI survivors continue to experience long-term physical, cognitive, and psychologic disorders and require extensive rehabilitation, which may disrupt relationships and prevent return to work. ${ }^{2}$ About $37 \%$ of people with mild TBI (mTBI) cases and $51 \%$ of severe cases were unable to return to previous jobs. A study examining psychosocial burden found that people with a history of TBI reported greater feelings of loneliness compared with individuals without TBI. ${ }^{3}$

Within the US military, the Defense and Veterans Brain Injury Center (DVBIC) indicates that $>417,503$ service members (SMs) have been diagnosed with TBI since November $2000 .{ }^{4}$ Of these, $82.4 \%$ were classified as having a mTBI, or concussion (Tables 1 and 2). The nature of combat and military training to which SMs are routinely exposed may increase the risk for sustaining a TBI. Specifically, the in- creased use of improvised explosives devices by enemy combatants in the recent military conflicts (ie, Operation Enduring Freedom, Operation Iraqi Freedom and Operation New Dawn) resulted in TBI being recognized as the signature injury of these conflicts and brought attention to the prevalence of concussion within the US military. ${ }^{5,6}$ In the military, the effects of concussion can decrease individual and unit effectiveness, emphasizing the importance of prompt diagnosis and proper management. ${ }^{7}$

Typically, patients recover from concussion within a few weeks of injury; however, some individuals experience symptoms that persist for months or years. Studies found that early intervention after concussion may aid in expediting recovery, stressing the importance of identifying concussion as promptly as possible. ${ }^{8,9}$ Active treatment is centered on patient education and symptom management, in addition to a progressive return to activities, as tolerated. Patient education may help validate the symptoms of some patients, as well as help to reattribute the symptoms to benign causes, leading to better outcomes. ${ }^{10}$ Since TBI is such a relevant health concern within the DoD, it is paramount for practitioners to understand what resources are available in order to identify and initiate treatment expeditiously.

This article focuses on the clinical tools used in evaluating and treating concussion, 
TABLE 1 Classification of Traumatic Brain Injury Severity ${ }^{18}$

\begin{tabular}{llll} 
Criteria & Mild & Moderate & Severe \\
\hline Structural imaging & Normal & Normal or abnormal & Normal or abnormal \\
\hline Loss of consciousness & $0-30$ min & $>30$ min and $<24 \mathrm{~h}$ & $>24 \mathrm{~h}$ \\
\hline Alteration of consciousness/mental state & A moment up to $24 \mathrm{~h}$ & $>24 \mathrm{~h}$; severity based on other criteria \\
\hline Posttraumatic amnesia & $0-1 \mathrm{~d}$ & $>1$ and $<7 \mathrm{~d}$ & $>7 \mathrm{~d}$ \\
\hline Glasgow Coma Scale (best available score in first $24 \mathrm{~h}$ ) & $13-15$ & $9-12$ & $<9$
\end{tabular}

aAlteration of mental status must be immediately related to the trauma to the head. Typical symptoms would be looking and feeling dazed and uncertain of what is happening, confusion, difficulty thinking clearly or responding appropriately to mental status questions, and being unable to describe events immediately before or after the trauma event.

and best practices treatment guidelines for health care providers (HCPs) who are required to evaluate and treat military populations. While these resources are used for military SMs, they can also be used in veteran and civilian populations. This article showcases 3 DoD clinical tools that assist HCPs in evaluating and treating patients with TBI: (1) the Military Acute Concussion Evaluation 2 (MACE 2); (2) the Progressive Return to Activity (PRA) Clinical Recommendation (CR); and (3) the Concussion Management Tool (CMT). Additional DoD clinical tools and resources are discussed, and resources and links for the practitioner are provided for easy access and reference.

\section{MILITARY ACUTE CONCUSSION EVALUATION 2}

Early concussion identification and evaluation are important steps in the treatment process to ensure timely recovery and return to duty for SMs. As such, DVBIC assembled a working group of military and civilian brain injury experts to create an evidence-based clinical practice guideline for the assessment and management of concussion in a military operational setting that could be learned and effectively used by corpsmen and combat medics in the battlefield to screen for a possible concussion. ${ }^{7}$ This team created the first version of the MACE, a clinical tool that prompted a systematic assessment of concussion related symptoms, neurologic signs, and cognitive deficits. The cognitive assessment portion was based on the standardized assessment of concussion (SAC) that had
TABLE 2 US Department of Defense Traumatic Brain Injuries Worldwide, 2000 to 2019

\begin{tabular}{lc} 
Traumatic Brain Injury Types & No. (\%) \\
\hline Penetrating & $5,388(1.3)$ \\
\hline Severe & $4,362(1.0)$ \\
\hline Moderate & $42,642(10.2)$ \\
\hline Mild & $343,980(82.4)$ \\
\hline Not classifiable & $21,131(5.1)$ \\
\hline Total & $\mathbf{4 1 7 , 5 0 3}$
\end{tabular}

been reported by McCrea and colleagues in 1998. ${ }^{11}$ Soon after its creation, field utilization of the MACE for screening of concussion was mandated by the Army through an All Army Action (ALARACT 178/2008) and for all of the Services through the DoD Instruction (DoDI) 6490.11 published in 2014. ${ }^{12}$

The MACE has been updated several times since the original version. Most recently, the MACE was revised in 2018 to include a vestibular oculomotor assessment section, and red flags that immediately alert the HCP to the need for immediate triage referral and treatment of the patient possibly at a higher echelon of care or with more emergent evaluation. ${ }^{13-15}$ Additionally, the neurologic examination was expanded to increase clarity and comprehensiveness, including speech and balance testing. Updates made to the tool were intended to provide a more thorough and informative evaluation of the SM with suspected concussion. 
TABLE 3 US Department of Defense Clinical Tools and Resources

\begin{tabular}{|c|c|c|}
\hline Resources & Type & Website \\
\hline MACE 2 & Clinical tool & dvbic.dcoe.mil/system/files/resources/MACE2.pdf \\
\hline MACE 2 Provider Training & Training materials & $\begin{array}{l}\text { dvbic.dcoe.mil/sites/default/files/MACE2_DVBIC_ProviderTraining } \\
\text { _04112019_508.pdf }\end{array}$ \\
\hline MACE 2 Instructor Guide & Training materials & $\begin{array}{l}\text { dvbic.dcoe.mil/sites/default/files/MACE2_DVBIC_InstructorGuide } \\
\text { _\%285002.1.1\%29508.pdf }\end{array}$ \\
\hline MACE 2 Student Workbook & Training materials & $\begin{array}{l}\text { dvbic.dcoe.mil/sites/default/files/MACE2_DVBIC_StudentWorkbook } \\
\text { _\%285005.1.1\%29508.pdf }\end{array}$ \\
\hline VOMS for Concussion Instructions & Training materials & dvbic.dcoe.mil/system/files/5004.1.1.21_DVBIC_VOMS_Visual_Guide_508.pdf \\
\hline СMT & Clinical tool & $\begin{array}{l}\text { dvbic.dcoe.mil/system/files/resources/4899.1.1.15_CMT_Pocket\%20Cards } \\
\text { _508v2.pdf }\end{array}$ \\
\hline CMT Provider Training & Training materials & dvbic.dcoe.mil/material/concussion-management-tool \\
\hline CMT Instructor Guide & Training materials & dvbic.dcoe.mil/sites/default/files/CMT_Instructor_Guide_5014.1.1.19_508.pdf \\
\hline CMT Student Workbook & Training materials & dvbic.dcoe.mil/sites/default/files/CMT_Student_Workbook_5015.1.1.27_508.pdf \\
\hline PRA CR for PCM & Clinical recommendation & dvbic.dcoe.mil/system/files/resources/1624.1.2.2_PRA_PCM_CR_508.pdf \\
\hline PRA CR for RP & Clinical recommendation & dvbic.dcoe.mil/system/files/resources/1626.1.2.2_PRA_Rehab_CR_508.pdf \\
\hline Clinical Support Tool for PCM & Clinical support tool & dvbic.dcoe.mil/system/files/resources/1625.1.2.15_PRA_PCM_CST_508.pdf \\
\hline Clinical Support Tool for RP & Clinical support tool & $\begin{array}{l}\text { https://dvbic.dcoe.mil/system/files/resources/1627.1.2.15_PRA_Rehab } \\
\text { _CST_508.pdf }\end{array}$ \\
\hline Training Slides for PCM & Training materials & $\begin{array}{l}\text { dvbic.dcoe.mil/system/files/resources/DVBIC_ProgressiveReturnActivity } \\
\text { _PrimaryCareManager_ProviderEd_Slides.pdf }\end{array}$ \\
\hline Training Slides for RP & Training materials & $\begin{array}{l}\text { dvbic.dcoe.mil/system/files/resources/DVBIC_ProgressiveReturnActivity } \\
\text { _Rehabiltation_ProviderEd_Slides.pdf }\end{array}$ \\
\hline Educational Brochure for PCM & Educational materials & $\begin{array}{l}\text { dvbic.dcoe.mil/system/files/resources/dvbic_4306_return-to-activity_brochure } \\
\text { _v1.1_2018-10-23.pdf }\end{array}$ \\
\hline Patient Educational Tool for RP & Educational materials & dvbic.dcoe.mil/system/files/resources/2013_PRA_Rehab_PES_FINAL.pdf \\
\hline $\begin{array}{l}\text { Management of Headache } \\
\text { Following mTBI for PCM }\end{array}$ & Clinical recommendation & $\begin{array}{l}\text { dvbic.dcoe.mil/system/files/resources/dvbic_4309_management-headaches } \\
\text {-mTBI_CR_v1.0_2017-08-09.pdf }\end{array}$ \\
\hline $\begin{array}{l}\text { Management of Sleep Distur- } \\
\text { bances Following mTBI for PCM }\end{array}$ & Clinical recommendation & dvbic.dcoe.mil/system/files/resources/4014.1.2.2_SleepCR_508.pdf \\
\hline $\begin{array}{l}\text { Assessment and Management of } \\
\text { Visual Dysfunction Associated with } \\
\text { mTBI }\end{array}$ & Clinical recommendation & dvbic.dcoe.mil/system/files/resources/4896.1.1.2_VisDys_CR_508.pdf \\
\hline $\begin{array}{l}\text { Assessment and Management } \\
\text { of Dizziness Associated mTBI }\end{array}$ & Clinical recommendation & dvbic.dcoe.mil/system/files/resources/4842.1.2.2_Dizziness_CR_508.pdf \\
\hline
\end{tabular}

DoDi 6490.11, 18 September 2012 Instruction www.esd.whs.mil/Portals/54/Documents/DD/issuances/dodi/649011p.pdf incorporating change 2 effective 26 November 2019

Abbreviations: CMT, Concussion Management Tool; CR, clinical recommendation; DoDi, US Department of Defense Instruction; MACE 2, Military Acute Concussion Evaluation 2; mTBI, mild traumatic brain injury; PCM, primary care managers; PRA, progressive return to activity; RP, rehabilitation professional; VOMS, vestibular/ocular-motor screening. 
This latest version, MACE 2, is designed to be used by any HCP who is treating SMs with a suspected or potential TBI, not just corpsmen and combat medics in theater. The MACE 2 is a comprehensive evaluation within a set of portable pocket cards designed to assist end-users in the proper triage of potentially concussed individuals. The DoD has specified 4 events that require a MACE 2 evaluation: (1) SM was in a vehicle associated with a blast event, collision, or roll over; (2) SM was within 50 meters of a blast; (3) anyone who sustained a direct blow to the head; or (4) when command provides direction (eg, repeated exposures to the events above or in accordance with protocols). ${ }^{12}$ Sleep deprivation, medications, and pain may affect MACE 2 results, in addition to deployment related stress, chronic stress, high adrenaline sustained over time, and additional comorbidities. This tool is most effective when used as close to the time of injury as possible but also may be used later (after 24 hours of rest) to reevaluate symptoms. The MACE 2 Instructor Guide, a student workbook, HCP training, and Vestibular/Ocular-Motor Screening (VOMS) for Concussion instructions can be found on the DVBIC website (Table 3).

\section{Description}

The MACE 2 is a brief multimodal screening tool that assists medics, corpsman, and primary care managers ( $\mathrm{PCMs}$ ) in the assessment and identification of a potential concussion (Figure 1). Embedded in the MACE 2 is the Standardized Assessment of Concussion (SAC), a well-validated sports concussion tool, and the VOMS tool as portions of the 2-part cognitive examination. The entirety of the tool has 5 sections: (1) red flags; (2) acute concussion screening; (3) cognitive examination, part 1; (4) neurologic examination; and (5) cognitive examination, part 2. The end of the MACE 2 includes sections on the scoring, instructions for International Classification of Diseases, Tenth Revision, TBI coding, and next steps following completion of the MACE 2. The latest version of this screening tool impacts TBI care in several noteworthy ways. First, it broadens the scope of users by expanding use to all medically trained personnel, allowing any provider to treat SMs in the field. Second, it combines state-ofthe-science advances from the research field and reflects feedback from end-users collected during the development. Last, the MACE 2 is updated as changes in the field occur, and is currently undergoing research to better identify end-user utility and usability.

\section{Screening Tools}

- Red Flags. The red flags section aids in identifying potentially serious underlying conditions in patients presenting with Glasgow Coma Scale (GCS) between 13 and 15. A positive red flag prompts the practitioner to stop administering the MACE 2 and immediately consult a higher level of care and consider urgent evacuation. While the red flags are completed first, and advancement to later sections of the MACE 2 is dependent upon the absence of red flags, the red flags should be monitored throughout the completion of the MACE 2. Upon completion of patient demographics and red flags, the remaining sections of the MACE 2 are dedicated to acute concussion screening.

- Acute Concussion Screening. The acute concussion screening portion consists of 4 sections: description of the incident; alteration of consciousness or memory; a "check all that apply" symptom inventory; and a patient history that includes concussions within the past 12 months, headache disorders, and/or behavioral health concerns. The final portion of the acute concussion screening section provides an algorithm to identify a positive or negative concussion screen. When a negative screen is identified, the user is prompted to prescribe a 24-hour rest period and follow up with the SM based on the guidance in the CMT. A positive screen warrants the user to continue administration of the MACE 2.

\section{Neurologic and Cognitive Examinations}

- Cognitive Exam Part 1. The initial cognitive examination is designed to assess orientation to time (eg, What is the day of the week, day of the month, the month, the year, and the timeof day?) as well as 


\section{FIGURE 1 Military Acute Concussion Evaluation $\left(\right.$ MACE 2) ${ }^{\mathrm{a}}$}
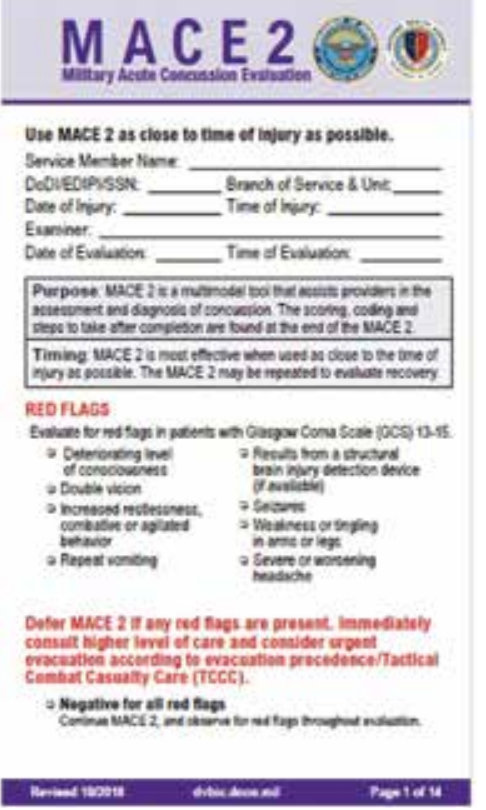

${ }^{a}$ Reprinted with permission of the Defense and Veterans Brain Injury Center. immediate recall of a short list of concrete words ( 5 words total, repeated for 3 trials). These tests are based on other neuropsychological measures designed to assess cognitive/mental status and short-term memory.

- The Neurological Exam. The neurological exam section of the MACE 2 includes brief neuropsychologic tests such as speech fluency and word finding. Other sections within the neurological exam assess the following: grip strength, vestibular function/balance (eg, tandem gait and single leg stance), as well as motor function (eg, pronator drift), autonomic nervous system function (eg, pupil response), and vestibular function (eye-tracking).

- Cognitive Exam Part 2. After completion of the first cognitive examination and the neurologic examination, the second part of the cognitive examination is initiated. Part 2 includes measures of short-term and working memory (eg, digits-reverse tasks, listing the months in reverse order, and a delayed recall task of the short list of concrete words presented in the first part). The final assessment is the administration of the VOMS, a tool developed from the sports concussion field and designed to measure vestibular-ocular function. ${ }^{13}$ It is critical to note that the VOMS is contraindicated if there is concern of an unstable cervical spine or absence of a trained HCP. An examination summary provides guidance on test scoring and yields a positive or negative indication for concussive injury. A positive test refers users to guidelines listed in the Concussion Management Tool for recommendations. The final page provides coding instructions for entering the results into the patient's electronic medical record for documentation and future reference.

\section{PROGRESSIVE RETURN TO ACTIVITIES CLINICAL RECOMMENDATION}

The Progressive Return to Activities Clinical Recommendation (PRA CR) also was developed by DVBIC for the DoD to assist military HCPs in managing SMs with concussion by providing systematic and evidence-based guidance to both prevent extended rest and promote return to full duty as quickly and safely as clinically indicated. The general guidance is to monitor the SM at each of the 6 stages in the process and safely and gradually increase activity to the next stage as tolerated. Daily symptoms are measured using the Neurobehavioral Symptom Inventory (NSI), which SMs self-administer every morning at each stage within the process.

Prior to initiation of the progressive return to activity, SM education using the educational brochure is strongly encouraged, as previous evidence suggests that it is an effective intervention during the acute stages of injury. ${ }^{10,11}$ Return to activity follows a 6 stage process, from stage 1 (rest) through stage 6 (unrestricted activity) (Table 4). Referral to rehabilitation providers (RPs) or higher care is left to the discretion of the PCM when (1) recovery is not progressing as anticipated; (2) progression is not being made within a 7-day period; or (3) symptoms worsen with time. The guidance outlined in the PRA CR is consistent with current policies and medical literature, and undergoes reviews as updates in the field emerge. The PRA for PCM, PRA for RP, Clinical Support Tool for PCM, Clinical Support Tool for RP, Training Slides for PCM, Training Slides for RP, Educational Brochure for PCM, and Patient Educational Tool for RP can be found on the DVBIC website (dvbic.dcoe.mil).

\section{Description}

To improve the clinical utility, 2 separate PRA CRs were developed specifically for PCMs (Figure 2) and RPs (Figure 3). The PRA CR for PCMs provides the initial framework to monitor SMs during recovery and gradually increase physical, cognitive, and vestibular/ balance activities as symptoms improve in order to return to preinjury activities. The PRA CR for RPs outlines the approach for treating SMs who meet 1 of the following criteria: recovery is not progressing as anticipated, there is no progression in 7 days, symptoms are worsening, the SM is symptomatic after exertional testing following 


\section{FIGURE 2 Progressive Return to Activity Clinical Recommendation Guidance for the Primary Care Manager in Deployed and Nondeployed Settings $s^{a, b}$}

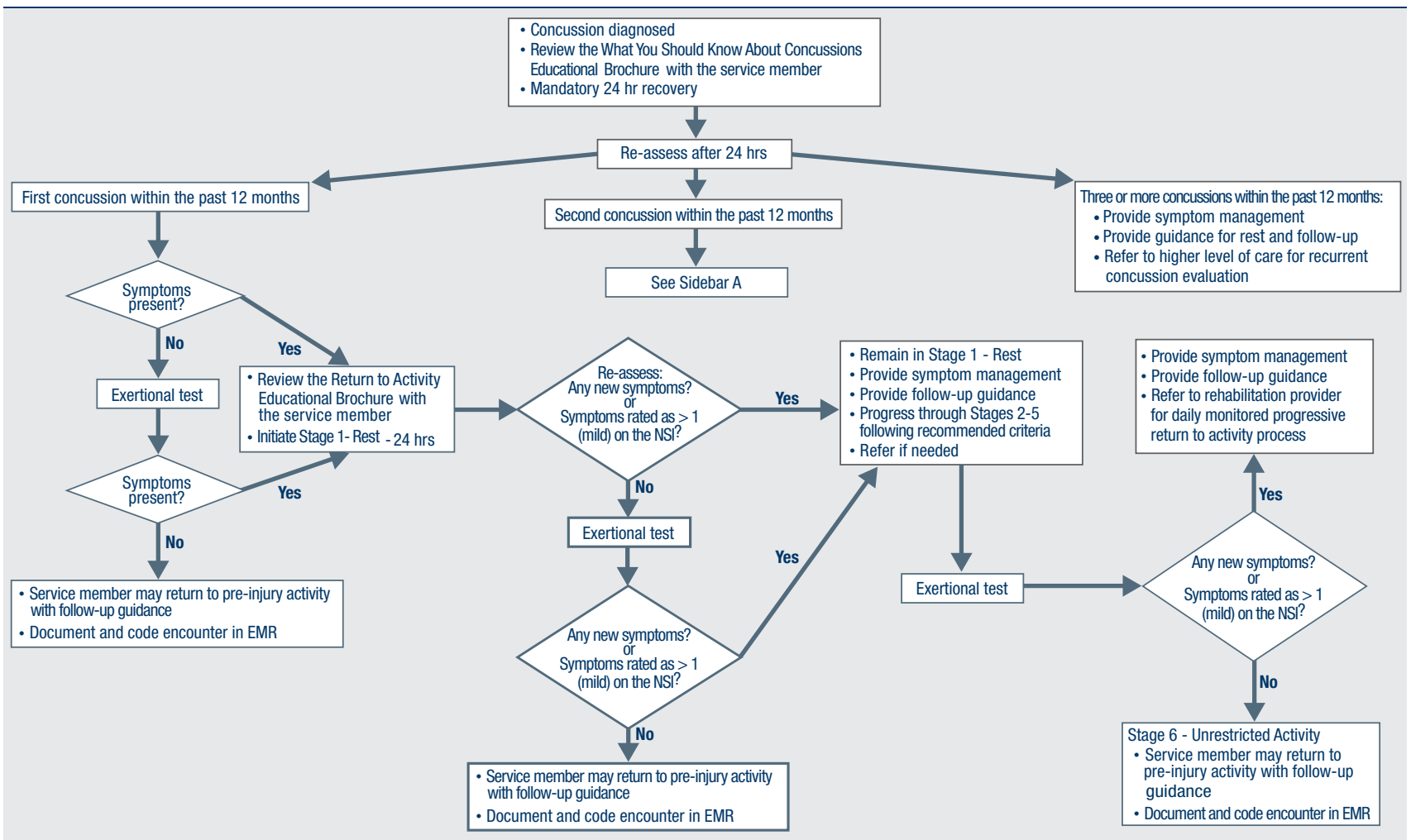

Abbreviations: EMR, electronic medical record; NSI, Neurobehavioral Symptom Inventory.

aFor Sidebar A, visit https://dvbic.dcoe.mil/system/files/resources/1624.1.2.2_PRA_PCM_CR_508.pdf.

${ }^{b}$ Reprinted with permission of the Defense and Veterans Brain Injury Center.

stage 5, or referral made per PCM judgment. Following the mandatory 24-hour rest period after a diagnosis of a concussion, progression through the PRA algorithm is based on history of concussion within the past 12 months (ie, 1,2 , or $\geq 3$ concussions) and symptomatology, with varying treatment pathways depending on the SM's responses to history and symptomology.

\section{Guidelines}

- One Concussion within Past 12 Months. Following the mandatory 24-hour rest period, if the SM is asymptomatic, then exertional testing (eg, activities such as push-ups, sit-ups, running in place, step aerobics, stationary bike, treadmill and/or hand crank) is performed at 65 to $85 \%$ of target heart rate for 2 minutes and symptoms are reassessed. If still asymptomatic, the SM may return to preinjury activity; however, if ex- ertional testing provokes symptoms $>1$ (mild) on the NSI, the SM should return to stage 1 with an additional 24 hours of rest. A second exertional test can be performed after stage 1 , and if symptoms are provoked, progression through the remaining stages 2 to 5 is encouraged. Symptoms are continually monitored throughout each stage to determine whether the SM is recovered sufficiently to proceed to the next stage.

- Two Concussion Within Past 12 Months. Following the mandatory 24-hour rest period, no exertional testing is performed, and SMs move directly into stage 1 and remain at stage 1 or stage 2 for 7 consecutive days with no symptoms $>1$ on the NSI before advancing through the remaining stages. Some defining features are longer rest periods (eg, 5 additional days of rest at stage 2) and additional patient education, symptom management, and follow-up. 
FIGURE 3 Progressive Return to Activity Following Acute Concussion/Mild Traumatic Brain Injury: Guidance for the Rehabilitation Provider in Deployed and Nondeployed Settings ${ }^{a}$

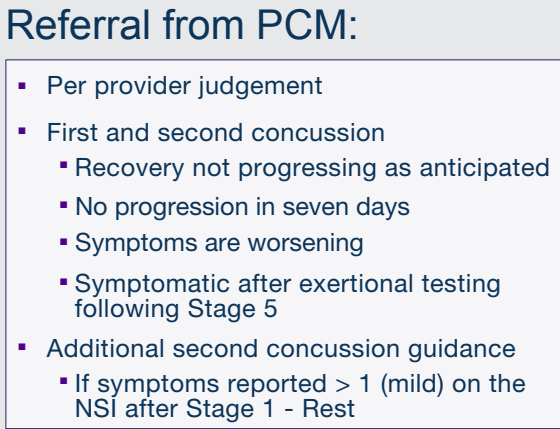

- Per provider judgement

- First and second concussion

- Recovery not progressing as anticipated

- No progression in seven days

- Symptoms are worsening

- Symptomatic after exertional testing following Stage 5

- Additional second concussion guidance - If symptoms reported $>1$ (mild) on the NSI after Stage 1 - Rest

Stage 3

Light Occupationoriented Activity

\section{Daily Assessment}

- NSI

- Resting HR

- Resting BP

- Consider medications, prior medical history and the possibilty of a previously undiagnosed condition

- If pre-injury NSI > 1, use clinical judgement

\section{Criteria for Progression}

- No new symptoms

- No symptoms $>1$ (mild) on the NSI

- Resting HR $<100$

- Resting BP < 140/90

* If second concussion in 12 months, service member stays at Stage 2 for a minimum of five days if above criteria are met
Stage 5

Intensive Activity

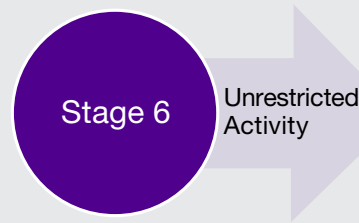

Stage 4

Moderate Activity

Abbreviations: BP, blood pressure; EMR, electronic medical record; HR, heart rate; NSI, Neurobehavioral Symptom Inventory; PCM, primary care manager.

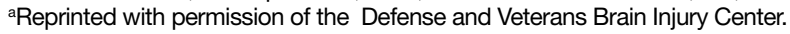

- Three or more Concussions Within Past 12 Months. Following the 24 hour mandatory rest period, in cases where $\geq 3$ concussions have occurred within a 12 month period, the recommendation is to provide guidance for symptom management rest and refer the SM to a higher level of care.

\section{Concussion Management Tool}

Beyond the initial assessment and concussion evaluation and the promotion of SMs' timely return to duty, the DoD developed a tool to help endpoint users manage concussion, to include those with more protracted symptoms (Figure 4). The CMT assists HCPs and the SMs they treat in the management of symptoms before and after they return to duty. Specifically, the CMT is designed to be given in combination with guidelines issued by the DoD in the PRA CR but extends management of concussion to include those symptoms experienced more long-term, or symptoms that are not solely addressed during the timeline of the PRA CR. Together, the MACE 2, PRA CR, and the CMT provide endpoint users with a set of tools to comprehensively evaluate, treat, and manage concussions in SMs.

\section{Description}

The CMT provides step-by-step guidance for the initial and comprehensive management of concussion, once a diagnosis is made using assessments in the MACE 2. All types of HCPs, particularly those with limited training, such as Navy Hospital Corpsman and Army Combat Medics, are the intended clinical audience for the CMT. This tool was revised in 2019 to better align with the MACE 2, PRA CR, and other DVBIC CRs, 
TABLE 4 Stages of the Progressive Return to Activity Process ${ }^{a}$

\begin{tabular}{lll} 
Stages & Type of Activity & Objectives \\
\hline 1 & Rest & Symptom resolution \\
\hline 2 & Light routine & Introduce and promote limited effort \\
\hline 3 & Light occupation-oriented & Increase light activities that require a combined use of physical, cognitive, and/or balance skills \\
\hline 4 & Moderate & Increase the intensity and complexity of physical, cognitive, and balance skills \\
\hline 5 & Intensive & $\begin{array}{l}\text { Introduce activity of duration and intensity that parallels the service member's typical role, function, } \\
\text { and tempo }\end{array}$ \\
\hline 6 & Unrestricted & Return to preinjury activities
\end{tabular}

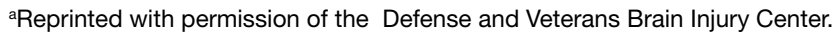

and replaces the 2012 Concussion Management Algorithm and the 2014 Army Concussion Management in Garrison Setting Algorithm. The first 2 sections of the CMT are action cards, which provide management guidelines for acute injuries up to 7 days following injury and for comprehensive management beyond 1 week. Guidelines within the CMT partially overlap with those in the PRA CR; however, the PRA is designed for a more acute timeline, whereas the CMT focuses on symptom management following a more protracted recovery. The CMT clinical tool, provider training, instructor guide, and student workbook all can be found on the DVBIC website (Table 3).

\section{DISCUSSION}

It is important for HCPs to have the skills and clinically relevant tools to optimize accurate TBI assessment. Early and accurate assessment and effective symptom management allows SMs to receive timely treatment based on clinical recommendations, and prevent and/or minimize secondary injury and prolonged recovery. Several longitudinal studies emphasize the benefits of early diagnosis and systematic follow-up. ${ }^{16-18}$ Prompt diagnosis, patient education, and early initiation to treatment may help optimize triage to care, mitigate prolonged symptoms by educating the patient on what to expect, and target specific symptoms early. ${ }^{8,10}$ Beyond the health outcomes of an individual SM, TBI recovery impacts unit readiness and consequently force readiness. As such, health outcomes and medical readiness are a priority of the Defense Health Agency (DHA).
The DHA priorities are, in part, based on DoD policy guidance for the management of concussion in the deployed setting. According to DoD instruction, "Medically documented $\mathrm{mTBI} /$ concussion in service members shall be clinically evaluated, treated, and managed according to the most current DoD clinical practice guidance for the deployed environment found in the Defense and Veterans Brain Injury Center (DVBIC) guidance, 'Medical Providers: Clinical Tools." 12 In 2018, the Deputy Secretary of Defense issued a memorandum regarding the comprehensive strategy and action plan for warfighter brain health. ${ }^{12}$ Therein, the memorandum acknowledges the enduring responsibility of the DoD to promote and protect the health and well-being of members of the nation's armed forces. Particular emphasis was placed on issuing a response to the effects caused by concussive impacts and exposure to blast waves. This response resulted in a commitment by the DoD to understanding, preventing, diagnosing, and treating TBI in all forms. Taken together, the message from the secretary of defense and instruction from the DoD is clear and makes imperative the use of DoD clinical tools to accomplish this commitment.

\section{CONCLUSIONS}

This article showcases 3 of the DoD's TBI clinical tools (MACE 2, PRA CR, and CMT) that assist HCPs in identifying and treating concussion. Over time, these tools undergo revisions according to the state of the science, and are adapted to meet the needs of clinicians and the SMs they treat. 
FIGURE 4 Concussion Management Tool Clinical Recommendation ${ }^{\mathrm{a}}$

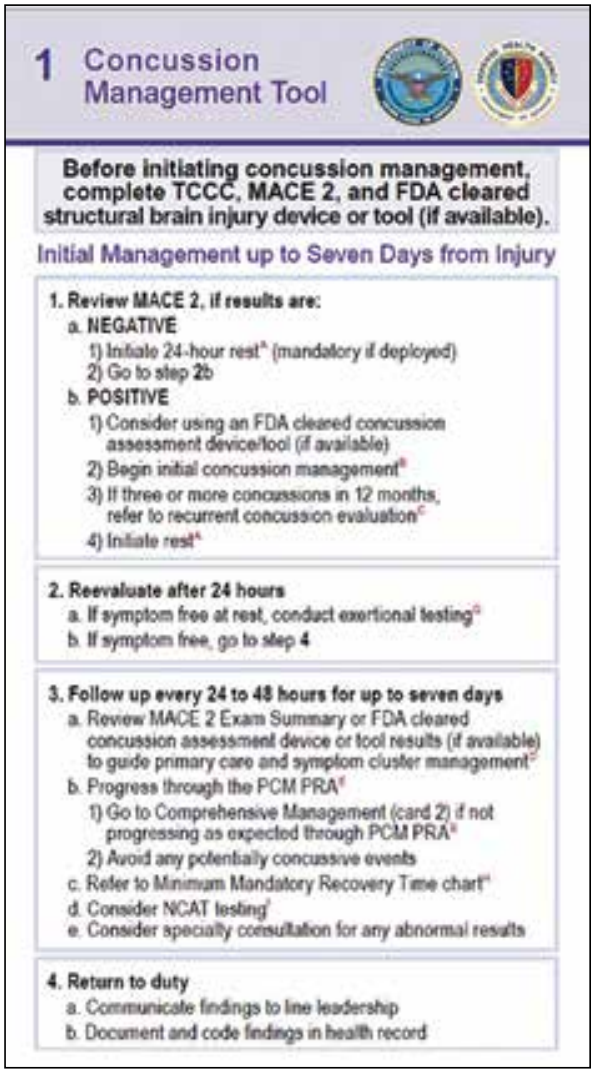

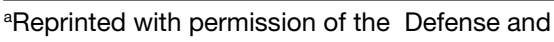
Veterans Brain Injury Center.
Studies are currently ongoing to better understand the effectiveness of these tools as well as to assist clinicians in making return-to-duty and/ or medical separation decisions. These tools assist clinicians throughout the recovery process; from initial assessment and treatment (acute phase), as well as with symptom management (acute and protracted symptoms).

Concussion is not a homogenous condition and the experiences of the SM, including events that may cause emotional distress, other injuries and/or other factors, may further complicate the injury. Accordingly, there is no single clinical tool that can conclusively determine return-to-duty status; rather, these tools can help characterize injury, validate, and treat symptoms, which have been suggested to improve outcomes. More research and data are needed confirm the effectiveness of these tools to improve outcomes.

It is beyond the scope of this article to provide a more in-depth discussion on TBI prevention or longer term effects/care. However, there are additional, personalized tools for specific symptoms, deficits, or dysfunctions following concussion. These tools include the Management of Headache Following mTBI for PCM CR, Management of Sleep Disturbances Following mTBI for PCM CR, Assessment and Management of Visual Dysfunction Associated with mTBI $\mathrm{CR}$, and Assessment and Management of Dizziness Associated mTBI CR. These tools enable endpoint users to evaluate and treat SMs as well as know when to elevate to higher levels of care.

The DoD commitment toward treating TBI influenced the development of the clini- cal tools highlighted in this article. They are the result of collective efforts among military and civilian TBI subject matter experts, data from medical literature and state-of-thescience research, and feedback from endpoint users to create the most effective, evidencebased tools. These tools undergo continuous review and revision to ensure alignment with the most up-to-date science within the field, to meet the needs of SMs and to continue the commitment to DoD concussion care.

\section{Acknowledgments}

This work was prepared under Contract (HT0014-19-C-0004) General Dynamics Information Technology and (W81XWH16-F-0330) Credence Management Solutions, and is defined as U.S. Government work under Title 17 U.S.C.\$101. Per Title 17 U.S.C.\$105, copyright protection is not available for any work of the U.S. Government. For more information, please contact dha.DVBICinfo@mail.mil.

\section{Author disclosures}

The authors report no actual or potential conflicts of interest with regard to this article.

\section{Disclaimer}

The opinions expressed herein are those of the authors and do not necessarily reflect those of Federal Practitioner, Frontline Medical Communications Inc., the US Government, or any of its agencies.

\section{References}

1. Centers for Disease Control and Prevention. Surveillance report of traumatic brain injury-related emergency department visits, hospitalizations, and deaths. https://www.cdc .gov/traumaticbraininjury/pdf/TBI-Surveillance-Report -FINAL_508.pdf. Published 2014. Accessed August 18, 2020.

2. Stocchetti N, Zanier ER. Chronic impact of traumatic brain injury on outcome and quality of life: a narrative review. Crit Care. 2016;20(1):148. Published 2016 Jun 21. doi:10.1186/s13054-016-1318-1

3. Kumar RG, Ornstein KA, Bollens-Lund E, et al. Lifetime history of traumatic brain injury is associated with increased loneliness in adults: A US nationally representative study. Int J Geriatr Psychiatry. 2020;35(5):553-563. doi:10.1002/gps.5271

4. Defense and Veterans Brain Injury Center. Worldwide DoD numbers for traumatic brain injury. 2020; https://dvbic dcoe.mil/sites/default/files/tbi-numbers/DVBIC _WorldwideTotal_2000-2019.pdf. Updated March 10, 2020. Accessed August 18, 2020.

5. Kennedy JE, Lu LH, Reid MW, Leal FO, Cooper DB. Correlates of depression in U.S. military service members with a history of mild traumatic brain injury. Mil Med. 2019;184(suppl 1):148-154. doi:10.1093/milmed/usy321

6. Marshall KR, Holland SL, Meyer KS, Martin EM, Wilmore $\mathrm{M}$, Grimes JB. Mild traumatic brain injury screening, diagnosis, and treatment. Mil Med. 2012;177(suppl 8):67-75. doi:10.7205/milmed-d-12-00110

7. French L, McCrea M., Baggett M. The Military Acute Concussion Evaluation. J Spec Oper Med. 2008;8(1):68-77. https://www.jsomonline.org/Publications/2008168French .pdf. Accessed August 18, 2020.

8. Kontos AP, Jorgensen-Wagers K, Trbovich AM, et al. Association of time since injury to the first clinic visit with recovery following concussion. JAMA Neurol. 2020;77(4):435-440. doi:10.1001/jamaneurol.2019.4552

9. Ponsford J, Willmott C, Rothwell A, et al. Impact of early 
intervention on outcome following mild head injury in adults. J Neurol Neurosurg Psychiatry. 2002;73(3):330-332. doi:10.1136/jnnp.73.3.33010.

10. Mittenberg W, Canyock EM, Condit D, Patton C. Treatment of post-concussion syndrome following mild head injury. J Clin Exp Neuropsychol. 2001;23(6):829-836. doi:10.1076/jcen.23.6.829.1022

11. McCrea M, Kelly JP, Randolph C, et al. Standardized assessment of concussion (SAC): on-site mental status evaluation of the athlete. J Head Trauma Rehabil. 1998;13(2):27-35. doi:10.1097/00001199-199804000-00005

12. US Department of Defense. Department of Defense Instruction, Number 6490.11 . Policy guidance for management of mild traumatic brain injury/concussion in the deployed setting. https://www.esd.whs.mil/Portals/54 /Documents/DD/issuances/dodi/649011p.pdf. Updated November 26, 2019. Accessed August 18, 2020.

13. Mucha A, Collins MW, Elbin RJ, et al. A brief Vestibular/ Ocular Motor Screening (VOMS) assessment to evaluate concussions: preliminary findings. Am J Sports Med. 2014;42(10):2479-2486. doi:10.1177/0363546514543775
14. Defense and Veterans Brain Injury Center. Military Acute Concussion Evaluation 2 (MACE 2). https://dvbic.dcoe.mil /material/military-acute-concussion-evaluation-2-mace-2. Updated August 18, 2020. Accessed August 18, 2020.

15. US Department of Defense, Defense Health Agency. Defense and Veterans Brain Injury Center releases new concussion screening tool. https://www.health.mil/News /Articles/2019/03/15/Defense-and-Veterans-Brain-Injury -Center-releases-new-concussion-screening-tool. Published March 15, 2019. Accessed August 18, 2020.

16. Schwab K, Terrio HP, Brenner LA, et al. Epidemiology and prognosis of mild traumatic brain injury in returning soldiers: a cohort study. Neurology. 2017;88(16):1571-1579. doi:10.1212NNL.0000000000003839

17. Mac Donald CL, Johnson AM, Wierzechowski L, et al. Outcome trends after US military concussive traumatic brain injury. J Neurotrauma. 2017;34(14):2206-2219. doi:10.1089/neu.2016.4434

18. Andelic N, Howe El, Hellstrøm T, et al. Disability and quality of life 20 years after traumatic brain injury. Brain Behav. 2018;8(7):e01018. doi:10.1002/brb3.1018 